\title{
Approaches to Accounting and Prediction of Fast Neutron Fluence on VVER Pressure Vessels for Estimation of RPV Residual Lifetime in Compliance with Russian Utility's Procedure
}

\author{
Gennady Borodkin ${ }^{1, a}$, Pavel Borodkin ${ }^{1}$, Nikolay Khrennikov ${ }^{1}$, Yuriy Ryabinin ${ }^{2}$, and Valeriy Adeev ${ }^{3}$ \\ ${ }^{1}$ Scientific and Engineering Centre for Nuclear and Radiation Safety (SEC NRS), 107140 Moscow, \\ Russia \\ ${ }^{2}$ Rosenergoatom Concern OJSC, Ferganskaya str., 25, 109507 Moscow, Russia \\ ${ }^{3}$ Kola NPP, Poliarnie Zori, Russia
}

\begin{abstract}
The Paper describes a new Russian Utility's regulatory document (RD EO) which has been recently developed and implemented since the beginning of 2013. This RD EO includes the procedure of RPV FNF monitoring and provides recommendations on how to predict fluence over the design lifetime taking into account results of FNF monitoring. The basic method of RPV neutron fluence monitoring is neutron transport calculations of FR in the vicinity of the RPV. Reliability of the calculation results should be validated by ex-vessel neutron-activation measurements, which were performed during different fuel cycles with different core loadings including new types of fuel.
\end{abstract}

\section{Introduction}

An important issue of reliable estimation of VVER non-restorable equipment lifetime, in particular, the reactor pressure vessel (RPV), is the prediction of radiation load parameters - fast neutron fluence (FNF) and fluence rate (FR). To ensure that the requirements of the Russian normative documents as regards to the accounting and prediction of RPV FNF for VVER type reactors are met, the Utility (Rosenergoatom Concern) has developed an in-house regulatory document (RD EO). The newly developed RD EO includes the procedure of RPV FNF monitoring and provides recommendations on how to predict fluence over the design lifetime taking into account results of FNF monitoring. The choice of calculation procedure should be done depending on separate type of VVER reactor designs. The criterion of such choice is reliable experimental validation of calculation procedures. Special attention has been focused on the neutron dosimetry measurements at an operating VVER reactor that provide experimental validation of the calculation procedure. The newly developed RD EO should be implemented at NPPs with different types of VVER reactors.

\footnotetext{
${ }^{a}$ Corresponding author: borodkin@secnrs.ru
}

This is an Open Access article distributed under the terms of the Creative Commons Attribution License 2.0, which permits unrestricted use, distribution, and reproduction in any medium, provided the original work is properly cited. 


\section{Requirements of the Russian Normative Documents}

Prediction of radiation load parameters (fluence, fluence rate, spectrum of neutrons) of reactor pressure vessels of Russian VVERs is regulated by the requirements of the Russian normative documents, in particular, by section 9.17 PNAE G-7-008-89, section 5.8 PNAE G-7-002-86, section 4.3.2.8 PNAE G-01-036-95, RB-007-99, RB-018-01 [1]. Compliance with these requirements is analyzed by expert analysis, carried out in SEC NRS.

The VVER RPV should be operated in accordance with the strict requirements of the Russian normative documents. Integrity of the VVER RPV should be ensured during the entire operation period of a NPP power unit.

In accordance with Russian normative documents, radiation load parameters of the VVER RPV are fluence of fast neutrons with energies above $0.5 \mathrm{MeV}$. FNF should be taken into account and predicted from the beginning of the NPP operation. Moreover, during a NPP unit operation a continuous monitoring of the RPV lifetime should be performed. Methods used for non-restorable equipment lifetime evaluation should be conservative. Distributions of FNF and FR on the RPV for different periods of operation should be presented in the NPP safety analysis reports (SAR).

Monitoring of the residual RPV lifetime should be performed by accounting and prediction of FNF in the critical points of RPV. The Utility organization (or NPP) should carry out monitoring and accounting of FNF. The account of FNF should be done using certified calculation procedures. It means that codes should be verified and validated against reliable measured data. Unified calculation procedures are recommended to be used for current FR monitoring at VVER RPVs. They should be tested by measurements. However, nowadays the Utility organization has no any sufficient tool which is appropriate to meet all regulatory requirements as far as the accounting and prediction of VVER RPV fast neutron fluence is concerned.

\section{Approaches to Accounting and Prediction of Fast Neutron Fluence}

To ensure that the requirements of the Russian normative documents [1] are met in part of accounting and prediction of RPV FNF for VVER type reactors, the Russian Utility's new regulatory document, RDEO 1.1.2.29.0913-2012 (RD EO) [2], has been recently developed and implemented since the beginning of 2013. Another purpose why such Utility's tool is necessary is that the design estimation of end-of-life FNF on the RPV cannot take into account real operation data (real core loadings, operating conditions) and may give redundant conservative estimates in the FNF prediction. On the other hand, RPV lifetime management is needed (in accordance with the new revision of PNAE G-7-008). One of ways of such management is to decrease RPV FNF by optimization of reactor core loadings, for example, by installation of burn-up fuel at the core periphery.

The newly developed procedure RD EO 0913-2012 regulates:

a. Calculation estimation of FNF on the RPV,

b. Experimental validation,

c. Prediction of FNF at the design end-of-life.

\subsection{Calculation Procedure and Approach to FNF Accounting}

The maximum neutron fluence for RPV critical points should be accounted over all period of operation. This should be done by NPP staff and held by calculation procedures. The account of FNF includes main steps such as:

- monitoring of FNF for previous cycles;

- prediction of FNF for the next cycle;

- correction of FNF after end of the cycle. 


\section{$15^{\text {th }}$ ISRD}

The basic method for RPV neutron fluence monitoring is a neutron transport calculation of FR in the vicinity of RPV. The choice of a calculation procedure should be oriented to separate type of VVERs. Certification of computer codes is the second necessary procedure in Russian practice of RPV fluence monitoring. New RD EO demands that computer codes used for safety justification are to be certified. It means that codes should be verified and validated by reliable measured data. Usually, a methodical uncertainty (without neutron source and material content uncertainty) of the codes, which perform FNF neutron transport calculations, is approximately $10 \%$.

Traditional features of a calculation model are a 60-degree sector of VVER, which includes: pinwise neutron source distributions in periphery assemblies; neutron source takes into account a mixture of $\mathrm{U} / \mathrm{Pu}$ fission spectrum; a variation of release of neutron per fission depending on burn-up. A new feature is neutron source preparation from an analysis of 360-degree neutron-physics core calculations. Earlier, the 30 and/or 60 degree core calculation results were used for core source model development. Also, RD EO recommends developing the neutron source as a combination of neutron physical core calculations and in-core power distribution measurements performed by a standard in-core monitoring system.

The account of FNF is taken by adding some margins to the calculation estimation of FNF. The value of margins depends on the validation results of the used calculation procedure.

\subsection{Experimental Validation of Calculation Procedure}

The criterion of reliability of calculation procedure is its experimental validation. Special attention is paid to the neutron dosimetry measurements that provide experimental validation of calculation procedure.

Ex-vessel experimental procedures are based on installation techniques developed by SEC NRS and recommended as normative document RB-018-01. A standard set of detectors and their characteristics are described in RB-018-01.

The neutron-activation measurements can provide measured data over the entire height of the reactor core. An azimuth angle of usually used neutron activation detector (NAD) installations is not less than 45 degrees. Irradiation of NAD is carried out over one fuel cycle. After that, measured FR should be compared with the calculated value. RD EO also regulates the frequency of such measurements. A period between routine measurements for each NPP unit should be not less than once in 6 years. Additional measurements are used for FR confirmation:

a) during the first three cycles from the beginning of operation - for check-up of the FR design values;

b) during reactor uprating (one cycle with operation at 104\%, 107\% etc.)

c) change in operation period:

- power unit lifetime extension (up to 45, 60 years);

- extension of the each fuel cycle (up to 16,18 months).

d) for the planning cycle, if the predicted FR is up 10\% from FR of previous cycles, which may result from

- changes in the design of the core or in-vessel structure;

- using new fuel types or new fuel assembly designs;

- using burn-up or high burn-up fuel assemblies at the periphery of core.

On average, the period between measurements corresponds to in-service inspection of RPV (once in 4 years in accordance with para. 8.2 of PNAE G-7-008-89). 
The criterion of the calculation method reliability is that the ratios of calculated and measured results do not exceed 1.15. When such criterion is achieved, the margin factor in the calculated estimation of FNF for a given cycle should be 1.15. In case the measurements for power unit with VVER are limited, greater margin factors should be taking into account. On the other hand, when the coincidence between the calculated and measured results exceeds $15 \%$, the developer of the calculation procedure should carry out additional analyses. The accounting of FNF should be done with the use of conservative margins up to $30 \%$.

\subsection{Prediction of FNF to Design Lifetime}

The prediction of FNF over the design lifetime is made by using the results of monitoring of FNF during previous cycles and predicted calculated values of the FNF for all planned cycles up to the end of the design lifetime. The prediction of FNF takes into account the real effective operation time for all completed fuel cycles. For the planned fuel cycles, the operation time is considered as the capacity factor of reactor power. The criterion of the FNF prediction is the comparison with the design fluence value $\overline{F_{\text {design }}}$. The prediction of FNF regulated by formula (1):

$$
F_{\tau}=\frac{F_{t}+\sum_{M} \boldsymbol{F}_{j}}{T_{N}+\frac{\sum_{M} \Delta t_{j}}{C F}} \cdot \tau \leq \overline{F_{\text {design }}},
$$

where:

$\mathrm{F}_{t}$ - is the actual FNF at the end of $\mathrm{N}$ cycle, which can be accounted for by:

$$
F_{t}=\sum_{N} F_{i}
$$

where:

$F_{i}-$ is the FNF accumulated during the i-th fuel cycle;

$\mathrm{N}-$ is the number of completed cycles, for which FNF is accounted for by monitoring with actual operation data;

$\mathrm{M}$ - is the number of planned cycles, for which FNF is calculated with the use of calculated input data;

$F_{j}$ - is the FNF accumulated over the planned cycle $j(j=N+1, \ldots, M)$;

$\mathrm{CF}$ - is the capacity factor set forth in the reactor design for FNF prediction to the end of life;

$\mathrm{T}_{\mathrm{N}}$ - is the calendar time to the beginning of $\mathrm{N}+1$ cycle;

$\Delta t_{j}-$ is the effective time of the $\mathrm{j}$-th cycle.

FNF is derived from the FR using the following equations:

$$
F_{i}=\Phi_{i} \cdot \Delta t_{i}, \quad F_{j}=\Theta_{j} \cdot \Delta t_{j},
$$

where:

$\Phi_{i}$ - is the fluence rate in the i-th cycle estimated using actual operation data;

$\Phi_{\mathrm{j}}$ - is the fluence rate in the $\mathrm{j}$-th cycle;

$\Delta \mathrm{t}_{\mathrm{i}}-$ is the effective time for the $\mathrm{i}$-th cycle.

In case of increasing the design value of FNF, further operation of the power unit is to be additionally decided on by the Utility taking into account the results of monitoring of FNF for a given power unit and paying special attention to the RPV lifetime management. 


\section{$15^{\text {th }}$ ISRD}

Table 1. Russian NPP units with VVER-440 and VVER-1000 undergoing ex-vessel dosimetry.

\begin{tabular}{|c|c|c|c|c|c|}
\hline & Unit 1 & Unit 2 & Unit 3 & Unit 4 & Unit 5 \\
\hline Kalinin NPP & $\operatorname{yes}(\times 2)$ & in progress & in progress & in progress & $-* *$ \\
\hline Novovoronezh NPP & shut down & shut down & yes $(\times 2)^{*}$ & yes $(\times 2)^{*}$ & yes $(\times 2)$ \\
\hline Kola NPP & yes $(\times 2)^{* *}$ & yes $(\times 2)^{*}$ & yes $(\times 2)$ & yes & $-* *$ \\
\hline Balakovo NPP & yes & yes $(\times 2)$ & yes $(\times 2)$ & yes $(\times 2)$ & $-* *$ \\
\hline Rostov NPP & yes & yes $(\times 2)$ & $-* *$ & $-* *$ & $-* *$ \\
\hline
\end{tabular}

\section{Application to the Routine FNF Accounting}

The newly developed RD EO should be implemented at NPPs with different types of VVER reactors and based on the verified and experimentally validated methods and procedures. Application of the approaches to FNF accounting and prediction is based on using unified calculation procedures and taking into account actual operation data.

\subsection{Calculation Procedure and its Validation}

The computerized procedure for VVER RPV FNF calculations - FASTER-440/1000 (Fluence

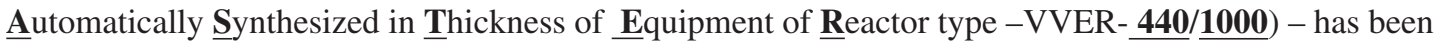
developed. The process is realized automatically in areas such as:

a) handling of input data from neutron physical calculations;

b) generating a core source for neutron transport calculations;

c) performing calculation by developed mathematically models;

d) computing FNF based on calculated estimation of FR.

Input data for generation of time-integrated neutron source distributions (used for DORT calculations) are usually based on a shift of fuel burn-up distributed over fuel assemblies during a fuel cycle. A neutron source associated with distribution of fuel burn-up shift in fuel assemblies corresponds to power density distribution over the reactor core during a fuel cycle.

Three-dimensional (3D) synthesis method based on results of DORT coupled with the BUGLE-96T library calculations is used for neutron fluence rate evaluations. The basic calculations were performed for 60-degrees symmetry sector. For modeling of the neutron source, the 3D total neutron release distribution was used. Two peripheral rows of assemblies were modeled taking into account pin-wise source distributions.

An analytical justification of the 3D synthesis method was carried out many times by comparison with precise 3D calculations based on Monte Carlo codes.

For validation of the calculated FR, ex-vessel measurements are used. SEC NRS is performing the ex-vessel measurements at all power units with VVER reactors. Russian NPP units which now undergo ex-vessel dosimetry are shown in Table 1.

\subsection{Implementation of the Approach at VVER-440}

The calculation procedure FASTER was suitably applied at the Kola NPP to account for and predict FNF during operation in the lifetime extension period.

Kola NPP includes now four power units with VVER-440s. The power units Nos. 1 and 2 pertain to the first generation of VVER-440 (V-230 design). The power units Nos. 3 and 4 are the second generation of the VVER-440 (V-213 design). By date, the first generation units of Russian NPPs have been in operation for approximately 10 years more than their design service lives. Life extension of longoperated VVER-440 of the second generation (design series V-213) is now also considered. During the 


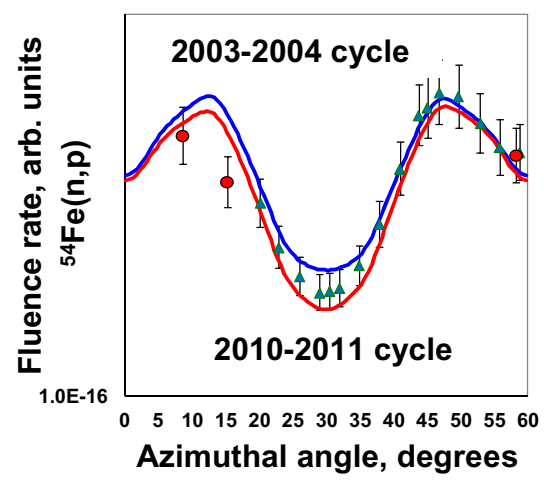

Figure 1. Comparison of FR distributions for Kola-2 (VVER-440/230) for different fuel cycles.

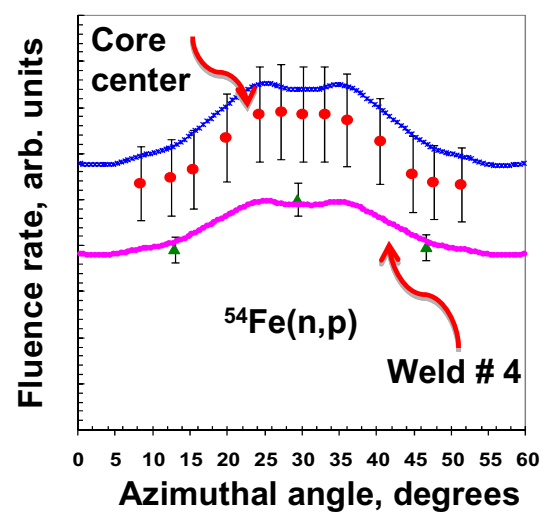

Figure 2. Comparison of FR distributions for Kola-4 (VVER-440/213).

lifetime extension process (started in 2000) it has been decided to pay more attention to monitoring of the FNF on the RPV.

Since 2004, the version of calculated procedure FASTER has been adapted to perform FNF calculations for VVER-440 (V-230 design). During lifetime extension of Units Nos. 3 and 4 the procedure was modified for application to the second generation of VVER-440 (V-213 design).

Neutron activation measurements in ex-vessel air cavity were carried out by SEC NRS at all power units of Kola NPP during different fuel cycles.

It should be mentioned that during operation in extended lifetime period, Kola NPP underwent modernization of core loadings in different fuel cycles at different power units. For the first generation of VVER-440s, new fuel types with recycled uranium and pin-by-pin profile throughout the fuel assembly were used.

The possibility to use profiled 2nd and 3rd-generation assemblies (FA-2 and FA-3, respectively) with $4.87 \%$ average enrichment and Gd rods was validated and it is now being implemented at Kola4 to uprate it up to $107 \%$. Gadolinium inside fuel assemblies improves average burn-up and reduces overall reactivity at the beginning of the fuel cycle.

Such changes in core loadings have resulted in the necessity of additional measurements in the exvessel cavity to confirm the reliability of FNF calculation procedure. New characteristics of modernized fuel assemblies were included in the calculation procedure to more accurately estimate FNF on the RPV.

Figures 1 and 2 show an example of the comparison of calculated and measured FR. 


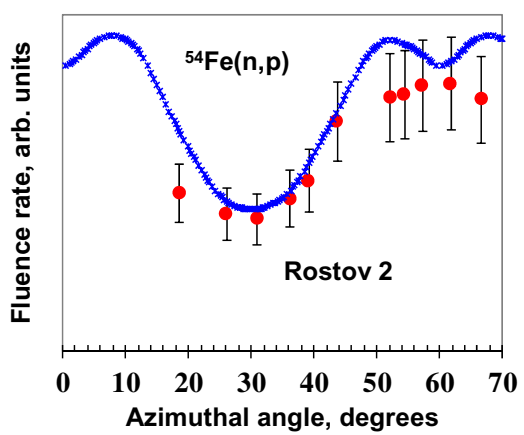

Figure 3. Comparison of FR azimutal distributions for Rostov-2 (second cycle, fresh TVS-2M assemblies).

A typical situation is that $\mathrm{C} / \mathrm{E}$ values for used threshold reactions of neutron activation detectors lie within the range of $\pm 10-15 \%$. The results of FR estimations show that calculation data are in agreement with measured ones. This fact confirms the reliability of the fluence calculation procedure and the possibility of using it for FNF calculations of RPV lifetime extension from 30 to 45 years. Nowadays, the FASTER procedure is used at Kola NPP also for optimization of reactor core loading in terms of reducing FNF at the end of the design lifetime. The main measures used are:

a) the use of dummy assemblies at Units 1 and 2:

- reduces FNF on RPV by up to 6 times,

- changes FNF maximum value in azimuthal distribution,

b) the use of burn-up and high burn-up assemblies in the positions closest to RPV (Units 3 and 4): reduces FNF on RPV by up to 2-3 times.

It has been shown that the use of the unified calculation procedure FASTER by Kola NPP staff allows to account and predict FNF on RPV and to manage the RPV lifetime.

\subsection{Implementation of the Approach at VVER-1000}

Validation of the calculation procedure was carried out in regard to NPP units with VVER-1000 type reactors. During ex-vessel measurements at Rostov-2, Balakovo-1, 2, 3 and 4, Kalinin-1 power units, modified type of fuel assemblies (TVS-2M, TVSA-PLUS) with extended fuel column were used. Moreover, nowadays VVER-1000 unites are operated in extended fuel cycles (up to 16-18 months) and with power uprate up to $104 \%$.

So the calculation procedure FASTER was validated by measurements for different conditions for operated power units:

- power: $100 \%$ and $104 \%$ of nominal;

- reactor types: VVER-1000/320, VVER-1000/338, VVER-1000/187;

- fuel assembly types: TVS-2, TVS-2M (canned and uncanned), TVSA, TVSA-PLUS, TVS-187;

- core loading: fresh periphery, burn-up assemblies, deep burn up assemblies;

Calculation-experimental validation was performed for all types of VVER-1000s, which are in operation and for all kinds of operating conditions, which could affect the RPV fast neutron fluence distribution. Figures 3, 4 and Table 2 demonstrate the comparison of calculated and measured fluence rates.

Results of VVER-1000 RPV neutron field parameters calculation-experimental investigations have shown good agreement of calculated and measured results. The $\mathrm{C}$ to $\mathrm{E}$ ratios lies in $10-15 \%$ for different 


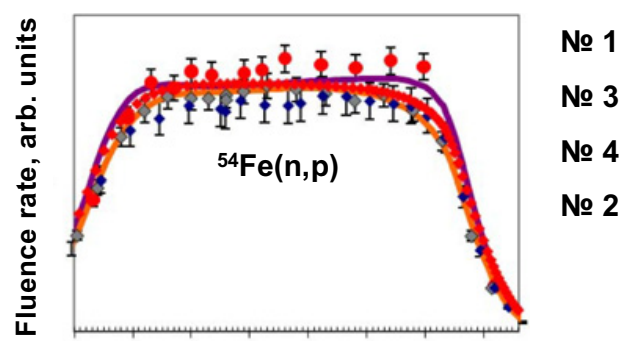

Height from core bottom, cm

Figure 4. Comparison of FR height distributions for Balakovo 1 - 4 power units (16 months, $104 \%$ ).

Table 2. Mean C/E values in ex-vessel positions for VVER-1000.

\begin{tabular}{|c|c|c|c|c|c|c|}
\hline Unit & VVER-1000 type & Cycle/ core & ${ }^{54} \mathrm{Fe}(\mathrm{n}, \mathrm{p})$ & ${ }^{58} \mathrm{Ni}(\mathrm{n}, \mathrm{p})$ & ${ }^{63} \mathrm{Cu}(\mathrm{n}, \alpha)$ & ${ }^{93} \mathrm{Nb}\left(\mathrm{n}, \mathrm{n}^{\prime}\right)$ \\
\hline Novoronezh-5 & 187 & Burn up fuel & 1.02 & 0.98 & 1.01 & 0.98 \\
\hline Balakovo 1 & 320 & $104 \%$ & 1.04 & 1.01 & - & - \\
\hline Balakovo 2 & 320 & 16 months & 1.01 & 1.05 & 1.07 & 0.95 \\
\hline Balakovo 3 & 320 & 15 months & 1.08 & 0.91 & 1.12 & 1.09 \\
\hline Balakovo 4 & 320 & $104 \%$ & 1.05 & 0.96 & 0.91 & 0.94 \\
\hline Rostov 2 & 320 & TVS-2M & 1.03 & - & 0.89 & 0.87 \\
\hline Kalinin 1 & 338 & TVSA-PLUS & 1.00 & 0.97 & 1.07 & 0.89 \\
\hline
\end{tabular}

positions of RPV. The latest investigations of VVER-1000 power units show new dependences for evaluation of FNF on RPV:

- first cycles of VVER (Rostov-2, Kalinin-4) operation could influence much FNF and the design value of FNF could be underestimated;

- the new types of fuel assemblies (TVS-2M) extend the reactor core axially by $15 \mathrm{~cm}$ that also increases FNF in the upper part of RPV (Welds 4, 5 and reactor support structure);

- the operation of the uprated reactor (104\% and 16 months cycle) affects the capacity factor in prediction of FNF at the end of lifetime.

It has been demonstrated that during any changes in reactor operating conditions or modernization of core loadings by using new types of fuel, additional monitoring of FNF at RPV should be performed. The latest calculations and measurements have approved the Russian Utility's requirements for accounting of FNF and predicting FNF over the design lifetime of RPV.

\section{Conclusion}

Account and prediction of FNF on the VVER RPVs is one of the main requirements of the Russian normative documents in terms of the RPV residual lifetime estimation. Approaches for accounting and prediction of FNF on VVER RPV are regulated now by recently developed Russian Utility's Procedure. Approaches are based on the latest calculation-experimental investigations, which have been performed for different power units during different fuel cycles. New computer-based procedure FASTER-440/1000 allows to perform FNF calculations in the vicinity of RPV of VVER. Ex-vessel neutron dosimetry measurements provide experimental validation of the calculation procedure on the operating VVER. The described Utility's document and developed calculation procedure allow monitoring and accounting of FNF and also predicting FNF down to the end of the design life taking into account results of FNF monitoring. 


$$
15^{\text {th }} \text { ISRD }
$$

\section{References}

[1] The List of Basic Legal Statements and Regulatory Standards used by Gosatomnadzor of Russia for State Regulation of Safety in the Field of Use of Atomic Energy. P-01-01-2011, Rostechnadzor, Moscow, Russia, 2011 (in Russian)

[2] The procedure for accounting and prediction of RPV fast neutron fluence for VVER type reactors. RD EO 1.1.2.29.0913-2012, Rosenergoatom Concern OJSC, Moscow, Russia, 2012 (in Russian)

[3] P.G. Borodkin, G.I. Borodkin, V.A. Adeev, J. Kohnheiser, Results of testing of calculation procedure for neutron fluence and spectrum on the VVER-440 RPV during operation with new fuel compositions, Proc. of 8-th International Scientific and Technical Conference "Safety Assurance of NPP with WWER", 28-31 May, 2013, Podolsk, Russia, 2013 